\title{
Navigation is Equal to Estimation by Eye and Palpation in Preventing Psoas Impingement in THA
}

\author{
Markus Weber MD, Michael Woerner MD, Benedikt Messmer Cand Med, \\ Joachim Grifka MD, Tobias Renkawitz MD
}

Received: 16 April 2016/ Accepted: 26 August 2016/Published online: 7 September 2016

(C) The Association of Bone and Joint Surgeons (B) 2016

\begin{abstract}
Background Iliopsoas tendon impingement is one possible reason for persistent groin pain and diminished functional outcome after THA. So far, estimation by eye and palpation is the standard procedure to intraoperatively assess the distance of the cup to the anterior rim. However, novel technologies such as imageless navigation enable intraoperative measurements of the cup in relation to the psoas tendon and bony landmarks.

Questions/purposes We asked whether psoas impingement (1) can be reduced using imageless navigation compared with the standard technique and (2) is associated with specific patient characteristics. Furthermore, we investigated (3) if anterior cup protrusion (overhang) is associated with lower outcome scores for pain and function.
\end{abstract}

\footnotetext{
The institution of one or more of the authors (MW, MW, BM, JG, TR) has received funding from the German Federal Ministry of Education and Research, project number 01EZ091.

All ICMJE Conflict of Interest Forms for authors and Clinical Orthopaedics and Related Research ${ }^{\circledR}$ editors and board members are on file with the publication and can be viewed on request. Each author certifies that his institution approved the human protocol for this investigation, that all investigations were conducted in conformity with ethical principles of research, and that informed consent for participation in the study was obtained.
}

Electronic supplementary material The online version of this article (doi:10.1007/s11999-016-5061-3) contains supplementary material, which is available to authorized users.

M. Weber $(\bowtie)$, M. Woerner, B. Messmer, J. Grifka,

T. Renkawitz

Department of Orthopedic Surgery, Asklepios Klinikum Bad

Abbach, Regensburg University Medical Center, Kaiser-Karl

V-Allee 3, 93077 Bad Abbach, Germany

e-mail: markus.weber@klinik.uni-regensburg.de
Methods The current study is a reanalysis of data from a randomized controlled trial evaluating navigation for THA; 135 patients were randomized for surgery with or without the use of imageless navigation. The risk for anterior protrusion of the cup above the acetabular rim and thus potential psoas impingement, defined as an overhang of the cup above the anterior acetabular rim as measured on postoperative $\mathrm{CT}$, was either controlled with the help of navigation or standard control by eye and palpation intraoperatively. Postoperatively, the anterior protrusion of the cup above the acetabular rim was measured on three-dimensional (3-D) CT by a blinded, external institute. In addition to patient-related outcome measures, the Harris hip score, Hip disability and Osteoarthritis Outcome Score, and EuroQol were obtained 1 year postoperatively. Altogether 123 data sets were available for primary analysis and 115 were available for 1-year followup.

Results There was no difference, with the numbers available, between the navigated and the control groups in terms of the mean distance of the cup below the anterosuperior acetabular rim $(3.9 \mathrm{~mm} ;-5.3$ to $12.6 \mathrm{~mm}$ versus $4.4 \mathrm{~mm} ;-7.9$ to $13.7 \mathrm{~mm} ; \mathrm{p}=0.72$ ) or the anteroinferior acetabular rim $(4.7 \mathrm{~mm} ;-6.2$ to $14.8 \mathrm{~mm}$ versus $4.2 \mathrm{~mm}$; -7.1 to $16.3 \mathrm{~mm} ; \mathrm{p}=0.29$ ). There was no difference, with the numbers available, in terms of the proportion of cups with anterior overhang (7\%, four of 57 versus, $15 \%, 10$ of $66 ; p=0.16)$, respectively. After controlling for potential confounding variables such as cup inclination, cup size, patient age, BMI, stage of arthritis, and length of skin incision, we found cup anteversion (hazard ratio [HR], $0.87 ; 95 \% \mathrm{CI}, 0.81-0.93 ; \mathrm{p}<0.001)$ and female sex (HR, 3.88; 95\% CI, 1.01-14.93; $\mathrm{p}=0.049$ ) were associated with a propensity to potential psoas impingement. With the numbers available, there were no differences observed in 
clinical scores between groups with and without anterior cup protrusion.

Conclusions We found no differences between imageless navigation and estimation by eye and palpation in preventing potential psoas impingement. Despite the comparable clinical outcome for patients with and without cup protrusion, the orthopaedic surgeon should be especially aware of propensity for psoas impingement in women.

Level of Evidence Level II, therapeutic study.

\section{Introduction}

Iliopsoas tendon impingement after THA is a potential source of groin pain and impaired function [3]. One study showed that $4.3 \%$ of patients had anterior psoas impingement develop after THA [1]. After clinical, laboratory, and radiographic exclusion of diagnoses requiring immediate surgical intervention such as dislocation, fracture, or infection $[8,9,15]$, the diagnosis of psoas impingement should be considered in patients presenting with painful active hip flexion after THA [10]. In addition to the patient's history and physical examination, CT offers the possibility to observe a prominence of the acetabular component above the acetabular rim with contact to the anterior iliopsoas tendon causing irritation [5]. In addition, ultrasound-guided infiltration of the psoas tendon enables further confirmation of the diagnosis. Psoas impingement has various treatment options ranging from ultrasoundguided peritendinous injections, iliopsoas tenotomy and lengthening, to acetabular component revision [7]. Intraoperative oversizing, lateralization, and retroversion of the acetabular component may increase the risk of cup protrusion over the anterior rim with secondary irritation of the iliopsoas tendon [7, 12].

Minimally invasive surgical techniques with reduced incision lengths and less-extensive exposures are safe without greater operative complication or component malrotation rates [16]. However, with these approaches it is more difficult for the orthopaedic surgeon to estimate the anatomic situation intraoperatively $[4,25]$. Thus, observation of a cup overhang above the anterior acetabular rim as commonly performed during THA might be compromised. In contrast, imageless navigation systems without the need for preoperative or intraoperative image acquisition and exposure to radiation have been reported to increase precision in positioning the acetabular component, achieve leg length and offset reconstruction, and prevent impingement $[19,24]$. The current study is a reanalysis of data from a randomized controlled trial [19]. The primary study dealt with ROM improvement by computer-assisted, functional optimization of cup position and containment. In the current analysis, we focused on propensity of psoas impingement as this novel navigation device is capable of detecting potential psoas impingement independently. During cup impaction, the system shows the distance to the previously registered iliopsoas tendon points. We compared this navigation-guided technique with the standard estimation of cup position by eye and palpation as usually performed in clinical practice. In terms of psoas impingement, other than various case reports $[5,6]$, one study described an association of psoas impingement to the difference between native acetabular and acetabular component version [13]. However, to the best of our knowledge, no randomized comparative trials are available that compare intraoperative techniques regarding their effect on prevention of potential psoas impingement in THA.

Our purposes therefore were to: (1) determine whether the use of imageless navigation can reduce the propensity of psoas impingement compared with standard intraoperative estimation by eye and palpation, (2) identify specific patient characteristics which predispose to psoas impingement, and (3) investigate if anterior cup protrusion (overhang) is associated with lower outcome scores for pain and function.

\section{Patients and Methods}

The current study is a reanalysis of data from a registered, prospective controlled trial (DRKS00000739, German Clinical Trials Register) evaluating navigation for THA. According to the main study, patients were randomized to with or without the use of imageless navigation [19]. The investigation was approved by the local ethics commission. The purpose of the primary study was to assess whether the artificial joint's ROM can be improved by computer-assisted, functional optimization of cup position and containment [19]. In addition to combined anteversion measurements [23], the navigation system independently detected protrusion of the cup above the anterior acetabular rim.

Between December 2011 and March 2013, a series of 783 patients admitted for primary uncemented THA at our institution owing to primary or secondary osteoarthritis was screened. According to the protocol of the main study [17, 19], eligible participants were patients between the ages of 50 and 75 years with an American Society of Anesthesiologists (ASA) score of 3 or below who were admitted for primary cementless unilateral THA attributable to primary or secondary osteoarthritis. Exclusion criteria were age younger than 50 years (as a postoperative CT scan was required) and older than 75 years (to ensure postoperative followup was achieved), 
ASA score greater than 3, arthritis attributable to hip dysplasia, posttraumatic hip deformities, and previous hip surgery. To allow gait analysis as intended for the primary outcome only patients who had no significant disease of the contralateral hip were included. Because of the strict inclusion criteria, of 783 patients screened, 597 did not meet the inclusion criteria. Twenty-seven patients declined to participate and 19 were excluded for other reasons (eg, cancellation of the operation owing to elevated inflammatory markers). The first five navigated cases were regarded as a learning curve. Finally, 135 patients were randomized to either navigated or standard THA. The randomization algorithm was performed as previously described [19].

THA was performed with all patients in the lateral decubitus position using a minimally invasive single-incision anterolateral approach by four experienced orthopaedic surgeons (JG, ES, MW, TR). Each surgeon had experience with more than 200 conventional and navigation-controlled THAs. Press-fit acetabular components and cement-free hydroxyapatite-coated stems (Pinnacle ${ }^{\circledR}$ cup, Corail ${ }^{\circledR}$ stem; DePuy, Warsaw, IN, USA) with metal heads of $32 \mathrm{~mm}$ were used. Of the initially 135 randomized patients, five $(3.7 \%$, four in the navigated and one in the control group) did not receive the allocated intervention. Of these five patients, one having shut down of the navigation system was included in analysis per intention to treat (ITT). Another eight patients $(5.9 \%$, six in the navigated and two in the control group) were excluded from analysis owing to missed or incorrect CT (four patients) or withdrawal of informed consent (four patients). Altogether 123 data sets were included for final CT analysis. For 1-year followup, 115 patients were available (53 in the navigated and 62 in the control group) (Fig. 1). Anthropometric characteristics of the navigation and control groups were comparable (Table 1).

For patients who were randomly allocated to the navigation group, the intraoperative distance of the cup to the anterosuperior and anteroinferior iliopsoas tendon was measured using imageless navigation (Hip 6.0 prototype; Brainlab, Feldkirchen, Germany). The registration process was performed as previously described [19]. After resection of the femoral head and exposure of the native acetabulum, acetabular osteophytes were removed. The acetabular rim and the psoas tendon anterosuperiorly and anteroinferiorly were registered. During cup impaction the navigation system showed the distance of the inserted cup to the psoas tendon according to the registered tendon points (Fig. 2). All surgeons aimed to avoid a protrusion of the cup above the anterior acetabular rim with contact to the iliopsoas tendon. The distance was shown continuously on the screen. For patients who were randomly allocated to the control group, intraoperative cup alignment in relation to the anterior acetabular rim was estimated by eye and palpation during cup impaction. Intraoperative characteristics of both intervention groups were comparable (Table 2).

At a mean of 6 weeks postoperatively, pelvic CT scans were performed (SOMATOM ${ }^{\circledR}$ Sensation 16 ; Siemens, Erlangen, Germany). Independent segmentation was obtained on the pelvic bone and the metal acetabular component by an independent external institute (Fraunhofer MEVIS, Bremen, Germany), blinded to individual patient data, including the type of surgical approach. Based on the segmented three-dimensional (3-D) bone models the distance of the cup in relation to the anterosuperior and anteroinferior acetabular rim was measured (Fig. 3). All measurements were performed with the leg in the neutral position. The accuracy of measuring anterior cup overhang on $\mathrm{CT}$ has been reported in previous studies [5, 13]. Negative values represent a protrusion of the cup above the acetabular rim resulting in a potential risk of psoas impingement. This was defined as propensity of iliopsoas impingement. Therefore, the results in the current study do not reflect clinically apparent psoas impingement but potential psoas impingement owing to an acetabular overhang. We then compared the proportion of patients with anterosuperior and anteroinferior protrusion of the acetabular component between the navigation-guided and the standard technique. In addition, we researched risk factors associated with potential psoas impingement. The anterior pelvic plane was used as the pelvic coordinate system for cup position. For clinical evaluation, patient-related outcome measures were obtained 1 year postoperatively using the validated Harris hip score (HHS), Hip Osteoarthritis Outcome Score (HOOS) [2], and EuroQol (EQ-5D ${ }^{\mathrm{TM}}$ ) [11].

An independent power calculation was performed for investigation of the primary endpoints in this secondaryoutcome analysis: protrusion of the cup above the anterosuperior and anteroinferior acetabular rim. Consequently, each of the corresponding hypotheses was tested on a Bonferroni-adjusted, two-sided 5\%/2 $=2.5 \%$ significance level. Owing to the lack of data regarding psoas impingement, we used previous results of navigationguided biomechanical leg length and offset reconstruction and conservatively set the effect size to 0.6 [24]. Based on these considerations, a sample size of 45 in each group achieved a power of $80 \%$ using two-sample t-tests (nQuery Advisor 7.0; Statistical Solutions Ltd, Cork, Ireland). Secondary endpoints, risk factors associated with psoas impingement, were tested on a 5\% significance level.

For statistical analysis, CT measurements are presented as median (range). Group comparisons were performed by Mann-Whitney U tests owing to the nonnormal data distribution. Absolute and relative frequencies were given for 


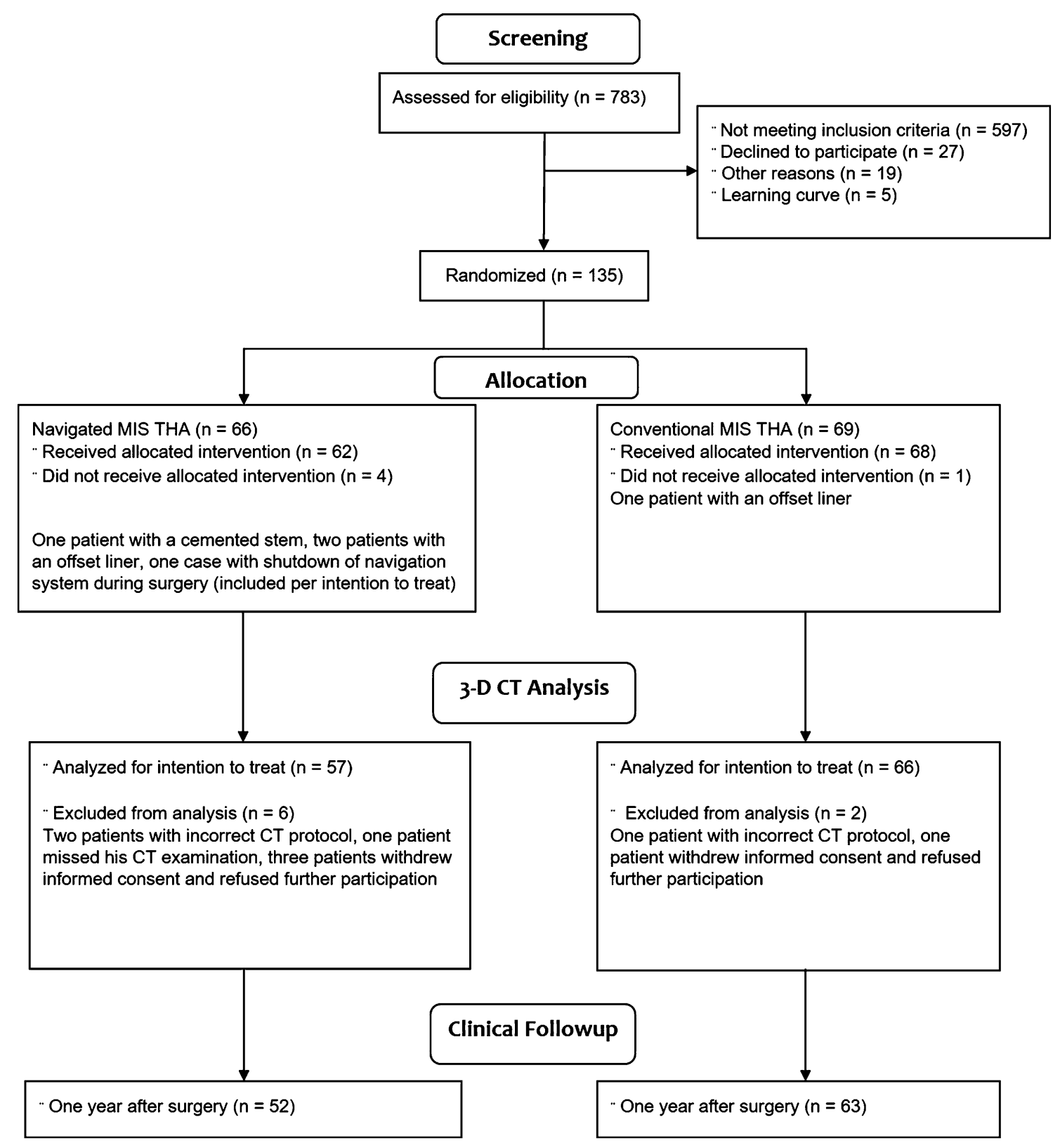

Fig. 1 The flow diagram shows the distribution of all participants in the study. MIS = minimally invasive surgery; $3-\mathrm{D}=$ three-dimensional.

categorical data and compared between study groups by chi-square tests. Multivariate logistic regression, including cup inclination, cup anteversion, sex, BMI, age, cup size, Kellgren-Lawrence score, and length of skin incision, was performed to search for risk factors associated with potential psoas impingement. The primary hypothesis was tested on a Bonferroni-adjusted two-sided $2.5 \%$ significance level, whereas all secondary hypotheses were tested in an explorative manner on a two-sided 5\% significance level. SPSS Statistics, Version 22.0 (IBM Corporation, Armonk, NY, USA) was used for analysis. All analyses were performed by intention to treat.

\section{Results}

With the numbers available, there was no difference between the navigated and standard methods for mean distance between the cup and the anterosuperior or anteroinferior acetabular rim. CT measurements showed a mean distance between the cup and the anterosuperior acetabular rim of $3.9 \mathrm{~mm}$ ( -5.3 to $12.6 \mathrm{~mm})$ in the navigated group and of $4.4 \mathrm{~mm}(-7.9$ to $13.7 \mathrm{~mm})$ in the control group $(\mathrm{p}=0.72)$, respectively (negative values $=$ cup above the anterior acetabular rim, positive values = cup below the anterior acetabular rim). Similarly, we found a 
Table 1. Anthropometric characteristics of the study group*

\begin{tabular}{lll}
\hline Characteristic & Control group & Navigated group \\
\hline Age (years) & $63 \pm 8$ & $63 \pm 8$ \\
Gender (men/women) & $33 / 33$ & $24 / 33$ \\
BMI $\left(\mathrm{kg} / \mathrm{m}^{2}\right)$ & $27 \pm 4$ & $27 \pm 4$ \\
Treatment side (right/left) & $39 / 27$ & $28 / 29$ \\
ASA Class 1 & 16 & 9 \\
ASA Class 2 & 30 & 33 \\
ASA Class 3 & 20 & 15 \\
Kellgren-Lawrence score & $9(5-10)$ & $8(6-10)$ \\
\hline
\end{tabular}

*Categorical data values are given as relative and absolute frequencies; quantitative data values are given as mean (SD) or median (range); ASA = American Society of Anesthesiologists.

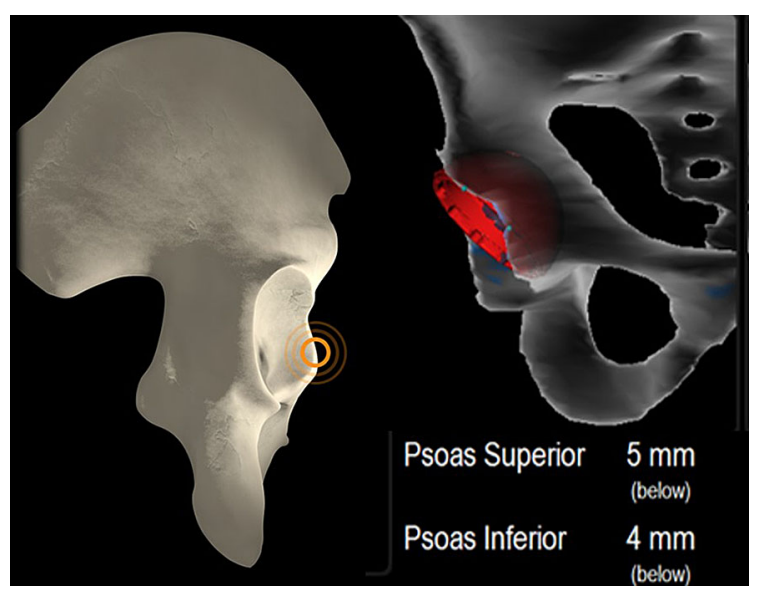

Fig. 2 In the navigation group the distance of the acetabular component in relation to the registered psoas tendon was visible on the screen.

Table 2. Intraoperative characteristics of the study group*

\begin{tabular}{lll}
\hline Variable & Control group & Navigated group \\
\hline Length of skin incision $(\mathrm{cm})$ & $10 \pm 1$ & $10 \pm 1$ \\
Operating time (minutes) & $64 \pm 14$ & $72 \pm 12$ \\
Cup size & $54(48-60)$ & $54(48-62)$ \\
Cup inclination $\left(^{\circ}\right)$ & $42 \pm 6$ & $43 \pm 5$ \\
Cup anteversion $\left(^{\circ}\right)$ & $18 \pm 9$ & $18 \pm 7$ \\
Cup coverage $(\%)$ & $90(52-99)$ & $88(66-100)$ \\
\hline
\end{tabular}

*Categorical data values are given as relative and absolute frequencies; quantitative data values are given as mean (SD) or median (range).

mean distance between the cup and the anteroinferior acetabular rim of $4.7 \mathrm{~mm}(-6.2$ to $14.8 \mathrm{~mm})$ in the navigated group and $4.2 \mathrm{~mm}(-7.1$ to $16.3 \mathrm{~mm})$ in the control group ( $p=0.29$ ) (Fig. 4). The percentage of patients with an anterosuperior and anteroinferior overhang of the cup

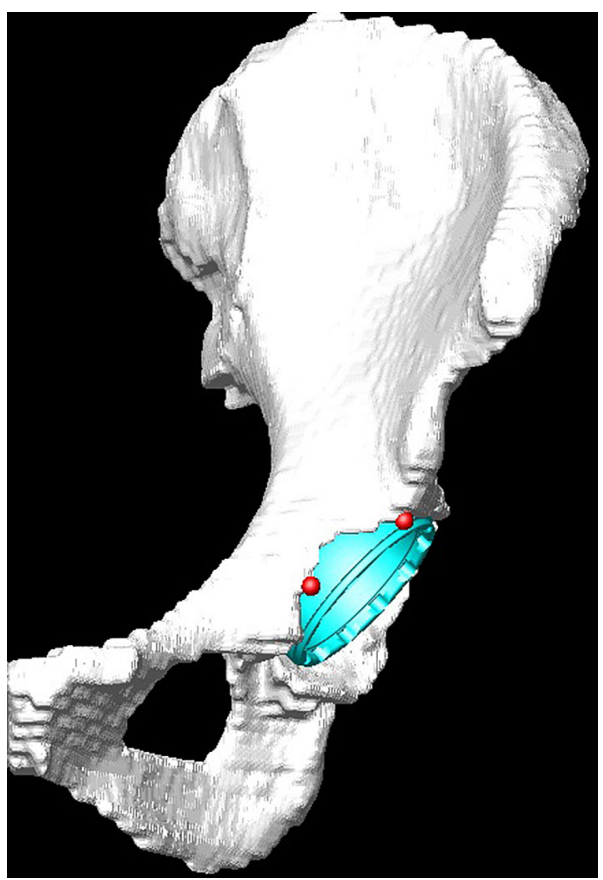

Fig. 3 Anterosuperior and anteroinferior distance of the cup to the anterior acetabular rim was measured on postoperative three-dimensional CT by a blinded external institute. The red dots represent the osseous anterosuperior and anteroinferior acetabular rim.

was $7.0 \%$ (four of 57) in the navigation group and $15.2 \%(10 / 66)$ in the visually controlled group $(\mathrm{p}=0.16)$ (Fig. 5).

Cup anteversion was associated with anterior overhang of the cup with a Spearman correlation coefficient of 0.46 $(\mathrm{p}<0.001)$ anterosuperiorly and 0.33 anteroinferiorly $(\mathrm{p}<0.001)$. Cup protrusion over the anterior acetabular rim was observed more frequently in women than in men (Supplemental Fig. 1. Supplemental material is available with the online version of $C O R R^{\circledR}$.). After controlling for potential confounding variables such as cup inclination, cup size, patient age, BMI, stage of arthritis, and length of skin incision, we confirmed cup anteversion (hazard ratio [HR], 0.87; 95\% CI, 0.81-0.93; p < 0.001) and female sex (HR, 3.88; 95\% CI, 1.01-14.93; p = 0.049) associated with propensity of potential psoas impingement (Table 3 ).

With the numbers available, there were no differences observed in clinical scores between groups with and without anterior cup protrusion. Mean HHS one year postoperatively was $96(50-100)$ for patients without potential psoas impingement compared with 96 (72-100) for patients with an acetabular overhang $(p=0.71)$. Similarly, we found HOOS values of 92.5 (48.8-100.0) in the group with cup protrusion and of 93.8 (63.8-99.4) in patients without cup protrusion $(\mathrm{p}=0.29)$. EQ-5D ${ }^{\mathrm{TM}}$ one year postoperatively showed excellent results independently of anterior cup overhang and thus potential psoas 


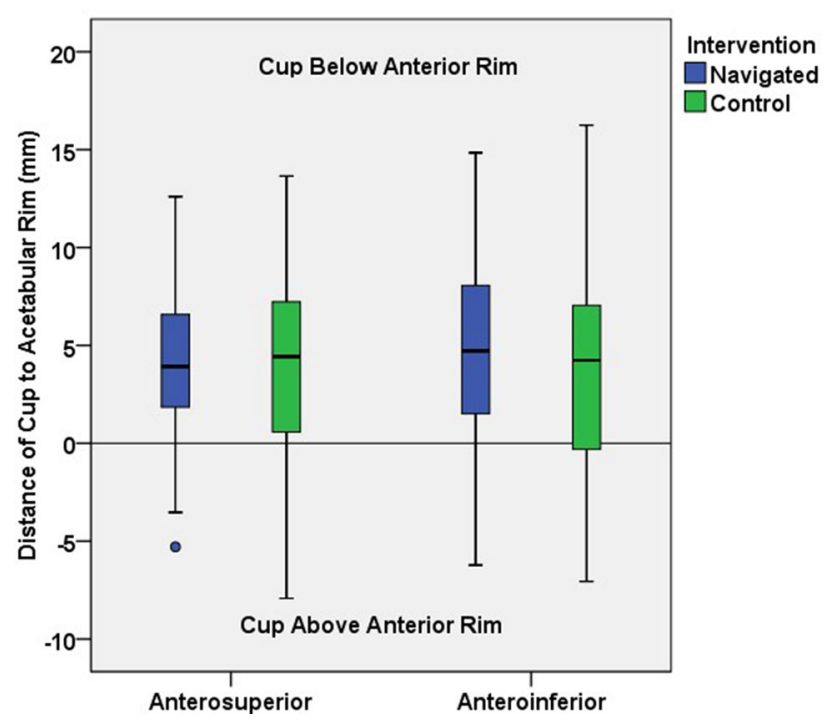

Fig. 4 The box and whisker plot compares the distance of the cup and the anterosuperior and anteroinferior acetabular rim between the navigation-guided and control group.

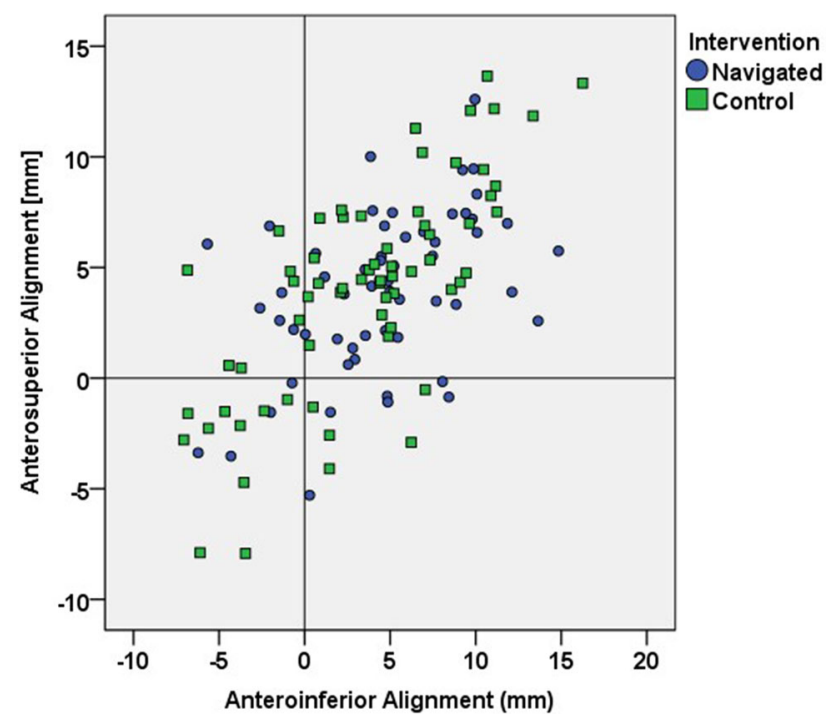

Fig. 5 The scatterplot shows the distribution of anterosuperior and anteroinferior cup alignment in relation to the anterior acetabular rim (negative values $=$ cup above the anterior acetabular rim, positive values $=$ cup below the anterior acetabular rim).

impingement with $1.0(0.79-1.00)$ versus $1.0(0.89-1.00)$ $(\mathrm{p}=0.96)$.

\section{Discussion}

Prior research has suggested psoas impingement to be a potential cause of pain and functional limitations after THA [3], therefore we believe it is important that surgeons
Table 3. Multivariate analysis of risk factors associated with propensity of psoas impingement

\begin{tabular}{llll}
\hline Variable & Hazard ratio & $95 \%$ CI & p value \\
\hline Cup inclination & 1.00 & $0.93-1.09$ & 0.91 \\
Cup anteversion & 0.87 & $0.81-0.93$ & $<0.001$ \\
Cup size & 1.00 & $0.81-1.24$ & 0.99 \\
Sex (female) & 3.88 & $1.01-14.93$ & 0.048 \\
Age & 0.98 & $0.93-1.04$ & 0.56 \\
BMI & 1.03 & $0.92-1.15$ & 0.60 \\
Kellgren-Lawrence score & 1.04 & $0.63-1.74$ & 0.88 \\
Length of skin incision & 0.88 & $0.62-1.25$ & 0.46 \\
\hline
\end{tabular}

are mindful of technique and patient factors that might be associated with it. However, to our knowledge, no studies have investigated these issues in a randomized trial. We therefore asked whether (1) the use of imageless navigation reduced the propensity of psoas impingement compared with intraoperative estimation by eye and palpation, (2) specific patient characteristics predisposed to psoas impingement, and (3) anterior cup protrusion (overhang) was associated with lower outcome scores for pain and function.

There are several limitations of this study. First, we measured overhang of the cup in relation to the acetabular anterior rim and did not account for symptomatic psoas impingement. Although the obtained distance is an objective outcome parameter, it primarily reflects radiographic findings suggestive of possible impingement and not clinical symptoms. Despite the lack of clinical problems attributable to psoas impingement in the current study group, the findings may be relevant as they reflect cup positions with a propensity of psoas impingement attributable to an anterior overhang. Second, the main study dealt with evaluation of an impingement-detection algorithm preventing osseous and prosthetic impingement following the concept of combined anteversion influencing cup position. However, the psoas impingement detection device of the navigation system separately restricted cup anteversion in relation to the psoas tendon. Third, $76 \%$ of the screened patients did not fulfill the inclusion criteria of the main study and thus had be excluded. This represents a potential selection bias. Therefore, the current results apply to patients with primary or secondary coxarthritis. According to the exclusion criteria the data do not include patients with posttraumatic hip deformities or hip dysplasia. In these cases, the results might be different owing to the anatomic variation. The use of navigation also has general limitations. First, especially in patients who are obese, pelvic landmarks can become obscured by overlying soft tissue, which can make direct referencing for 
computer-assisted surgery difficult $[18,21]$. Second, computers are susceptible to electronic failure, which happened once during our study. Therefore, surgeons using navigation always need to be aware of potential malfunctions in the system and should be able to continue operating without the assistance of a computer at any time. Additionally, we caution that our results might apply only to the particular prosthetic system used in this study. However, the use of a single device minimizes confounding factors related to design differences among implants.

To answer the first question of the study, imageless navigation and estimation by eye and palpation were capable of evaluating cup protrusion above the acetabular rim. The mean distance of the cup below the acetabular rim was 3 to $4 \mathrm{~mm}$ and thus comparable between both groups. To the best of our knowledge, no other comparative studies are available in the orthopaedic literature researching surgical techniques in terms of psoas impingement. Cadaveric analysis identified anatomic variances of the psoas tendon which might have an effect on psoas impingement [14]. CT-based calculations of psoas impingement areas found that the difference between native and final cup anteversion correlated with the radiographic severity of psoas impingement [13]. In the current study, no preoperative CT scans were available owing to strict radiation laws in our country. Therefore, we were not able to measure preoperative native acetabular version. In the current analysis, the percentage of patients in the navigated group with an anterior overhang of the cup (7\%) was half as much as the control group (15.2\%). Although the result was not statistically significant, this might have a clinically relevant effect. Therefore, further clinical trials are required assessing the effect of reduced anterior overhang as observed in our study on clinically symptomatic psoas impingement and outcome.

In terms of our second study aim, we performed a multivariate analysis to control for potential confounding variables associated with potential psoas impingement. A low cup anteversion resulted in a 1.15-fold increase of anterior acetabular cup protrusion. Interestingly we found female sex was associated with a 3.88-fold higher propensity of potential psoas impingement. Other suspected parameters like cup size, BMI, and incision length showed no correlation on propensity of potential psoas impingement. The unexpected association of female sex to potential psoas impingement might be attributable to slight differences between the male and female acetabulum. This sex-related anatomic variance is supported by a previous study showing a correlation between sex and acetabular diameter, anteversion, and depth of psoas valley [22]. In contrast, another study showed a predominance in males regarding higher impingement rates for prosthetic and osseous impingement [19]. Further studies found an association of native and final acetabular version [13] and head size [20] on psoas impingement.

Patient-related outcome measures were comparable between patients with and without an acetabular overhang. This might be because the maximum acetabular overhang was $7.9 \mathrm{~mm}$ anterosuperiorly and $7.1 \mathrm{~mm}$ anteroinferiorly. We found no prospective studies that have been published so far describing benchmarks for tolerable acetabular protrusion not predisposing to clinically symptomatic psoas impingement. Only one case series reported cup protrusion greater than $12 \mathrm{~mm}$ in patients with symptomatic psoas impingement whereas cup overhang was less than $8 \mathrm{~mm}$ in patients without psoas impingement [5]. In this context, only severely malpositioned cups with a higher extent of anterior overhang might result in clinically symptomatic psoas impingement. In addition, measurements of psoas impingement so far rely on static positions of the pelvis. As gait is a dynamic process, the distance of the psoas tendon to the anterior cup might vary during gait cycle and thus cannot be detected on CT.

In this randomized controlled trial analyzing the propensity of psoas impingement as measured on 3-D CT, we found no difference between estimation by eye and palpation versus navigation-guided minimally invasive THA. Future prospective studies focusing on symptomatic psoas impingement are required to identify benchmarks for anterior cup overhang causing psoas impingement. We found female sex was associated with cup protrusion over the anterior acetabular rim. Therefore, the orthopaedic surgeon should be especially aware of psoas impingement in women.

Acknowledgments We thank Ernst Sendter MD (Department of Orthopedic and Trauma Surgery, Vilsbiburg Hospital, Germany) for support as one of the senior surgeons in this study.

\section{References}

1. Ala Eddine T, Remy F, Chantelot C, Giraud F, Migaud H, Duquennoy A. [Anterior iliopsoas impingement after total hip arthroplasty: diagnosis and conservative treatment in 9 cases][in French]. Rev Chir Orthop Reparatrice Appar Mot. 2001;87:815819.

2. Blasimann A, Dauphinee SW, Staal JB. Translation, cross-cultural adaptation, and psychometric properties of the German version of the hip disability and osteoarthritis outcome score. $J$ Orthop Sports Phys Ther. 2014;44:989-997.

3. Browne JA, Polga DJ, Sierra RJ, Trousdale RT, Cabanela ME. Failure of larger-diameter metal-on-metal total hip arthroplasty resulting from anterior iliopsoas impingement. J Arthroplasty. 2011;26:978.e975-978.

4. Cheng T, Feng JG, Liu T, Zhang XL. Minimally invasive total hip arthroplasty: a systematic review. Int Orthop. 2009;33:14731481.

5. Cyteval C, Sarrabere MP, Cottin A, Assi C, Morcos L, Maury P, Taourel P. Iliopsoas impingement on the acetabular component: 
radiologic and computed tomography findings of a rare hip prosthesis complication in eight cases. J Comput Assist Tomogr. 2003;27:183-188.

6. Dalmau-Carola J. Iliopsoas impingement: a report of 2 cases. Pain Pract. 2014;14:457-460.

7. Dora C, Houweling M, Koch P, Sierra RJ. Iliopsoas impingement after total hip replacement: the results of non-operative management, tenotomy or acetabular revision. J Bone Joint Surg Br. 2007;89:1031-1035.

8. Grifka J, Keshmiri A, Maderbacher G, Craiovan B. [Clinical examination of the hip joint in adults][in German]. Orthopade. 2014;43:1115-1132; quiz 1132-1133.

9. Hube R, Dienst M, von Roth P. [Complications after minimally invasive total hip arthroplasty][in German]. Orthopade. 2014;43:47-53.

10. Jasani V, Richards P, Wynn-Jones C. Pain related to the psoas muscle after total hip replacement. J Bone Joint Surg Br. 2002;84:991-993.

11. Konig HH, Bernert S, Angermeyer MC. [Health Status of the German population: results of a representative survey using the EuroQol questionnaire][in German]. Gesundheitswesen. 2005;67:173-182.

12. Lequesne $\mathrm{M}$, Dang $\mathrm{N}$, Montagne $\mathrm{P}$, Lemoine A, Witvoet J. [Conflict between psoas and total hip prosthesis][in French]. Rev Rhum Mal Osteoartic. 1991;58:559-564.

13. Park KK, Tsai TY, Dimitriou D, Kwon YM. Three-dimensional in vivo difference between native acetabular version and acetabular component version influences iliopsoas impingement after total hip arthroplasty. Int Orthop. 2015 Nov 27. [Epub ahead of print]

14. Philippon MJ, Devitt BM, Campbell KJ, Michalski MP, Espinoza C, Wijdicks CA, Laprade RF. Anatomic variance of the iliopsoas tendon. Am J Sports Med. 2014;42:807-811.

15. Preininger B, Haschke F, Perka C. [Diagnostics and therapy of luxation after total hip arthroplasty][in German]. Orthopade. 2014;43:54-63.

16. Reininga IH, Zijlstra W, Wagenmakers R, Boerboom AL, Huijbers BP, Groothoff JW, Bulstra SK, Stevens M. Minimally invasive and computer-navigated total hip arthroplasty: a qualitative and systematic review of the literature. BMC Musculoskelet Disord. 2010;11:92.
17. Renkawitz T, Haimerl M, Dohmen L, Gneiting S, Wegner M, Ehret N, Buchele C, Schubert M, Lechler P, Woerner M, Sendtner E, Schuster T, Ulm K, Springorum R, Grifka J. Minimally invasive computer-navigated total hip arthroplasty, following the concept of femur first and combined anteversion: design of a blinded randomized controlled trial. BMC Musculoskelet Disord. 2011;12:192.

18. Renkawitz T, Haimerl M, Dohmen L, Woerner M, Springorum HR, Sendtner E, Heers G, Weber M, Grifka J. Development and evaluation of an image-free computer-assisted impingement detection technique for total hip arthroplasty. Proc Inst Mech Eng H. 2012;226:911-918.

19. Renkawitz T, Weber M, Springorum HR, Sendtner E, Woerner M, Ulm K, Weber T, Grifka J. Impingement-free range of movement, acetabular component cover and early clinical results comparing 'femur-first' navigation and 'conventional' minimally invasive total hip arthroplasty: a randomised controlled trial. Bone Joint J. 2015;97:890-898.

20. Rodriguez JA, Rathod PA. Large diameter heads: is bigger always better? J Bone Joint Surg Br. 2012;94(11 suppl A):52-54.

21. Sendtner E, Schuster T, Worner M, Kalteis T, Grifka J, Renkawitz T. Accuracy of acetabular cup placement in computerassisted, minimally-invasive THR in a lateral decubitus position. Int Orthop. 2011;35:809-815.

22. Vandenbussche E, Saffarini M, Taillieu F, Mutschler C. The asymmetric profile of the acetabulum. Clin Orthop Relat Res. 2008;466:417-423.

23. Weber M, Weber T, Woerner M, Craiovan B, Worlicek M, Winkler S, Grifka J, Renkawitz T. The impact of standard combined anteversion definitions on gait and clinical outcome within one year after total hip arthroplasty. Int Orthop. 2015;39:2323-2333.

24. Weber M, Woerner M, Springorum R, Sendtner E, Hapfelmeier A, Grifka J, Renkawitz T. Fluoroscopy and imageless navigation enable an equivalent reconstruction of leg length and global and femoral offset in THA. Clin Orthop Relat Res. 2014;472:31503158.

25. Worner M, Weber M, Lechler P, Sendtner E, Grifka J, Renkawitz $\mathrm{T}$. [Minimally invasive surgery in total hip arthroplasty: surgical technique of the future?][in German]. Orthopade. 2011;40:10681074. 